\title{
Adaptación psicométrica de la Escala de Factores Protectores (spf-24) en una muestra de sujetos colombianos
}

\author{
Psychometric Adaptation of the Scale of Protective Factors \\ (SPF-24) in a Sample of Colombian Subjects
}

\author{
Jose Ignacio Ruiz-Pérez ${ }^{1 \boldsymbol{\Delta}}$, Aura Nidia Herrera Rojas ${ }^{2}$
}

Universidad Nacional de Colombia

Fecha correspondencia:

Recibido: febrero 25 de 2018.

Aceptado: enero 22 de 2019.

Forma de citar:

Ruíz Pérez, J.I., \& Herrera Rojas, A.N.

(2019). Adaptación psicométrica de

la Escala de Factores Protectores

(SPF-24) en una muestra de sujetos colombianos. Rev.CES Psico, 12(2),

$1-11$.

\section{Open access}

(c) Copyright

Licencia creative commons

Ética de publicaciones

Revisión por pares

Gestión por Open Journal System

DOl: http://dx.doi.org/10.21615/

cesp.12.2.1

ISSN: 2011-3080

Sobre los autores:

1. Doctor en Psicología, Profesor Departamento de Psicología,

Universidad Nacional de Colombia.
Comparte

F日G.898 $\mathbb{R}^{\circ}$ 因

\section{Resumen}

Objetivo: Conocer el comportamiento psicométrico en sujetos colombianos de la Escala de Factores Protectores (SPF-24, de Ponce-García, Madewell, \& Kennison, 2015). Método: Muestra no aleatoria de n: 190 sujetos, entre 15 y 65 años, 51\% mujeres, respondieron a la SPF-24 y la Escala de Resiliencia Comunitaria (Ruiz, 2015). Resultados: Fiabilidad interna de la escala total de 0,93, y entre 0,83 y 0,86 por dimensiones. El análisis factorial exploratorio arrojó cuatro dimensiones relevantes: apoyo social, habilidades sociales, planificación y eficacia de metas. Las dos primeras corresponden a la estructura factorial del estudio original. También, a mayor resiliencia individual, más resiliencia comunitaria, sobre todo de afrontamiento comunitario. Discusión y conclusiones: La escala muestra índices satisfactorios de fiabilidad interna, de validez de constructo y de validez concurrente con medidas de resiliencia comunitaria. Es necesaria más investigación sobre la validez predictiva y concurrente del SPF-24.

Palabras clave: Resiliencia Individual, Resiliencia Comunitaria, Apoyo Social, Habilidades Sociales, Planificación, Eficacia de Metas.

\section{Abstract}

Objetive: To know psychometric behavior in a sample of Colombian individuals of the Scale of Protective Factors (SPF-24, Ponce-García, Madewell, \& Kennison, 2015). Method: a non-randomized sample (n: 190), aged between 15 and 65 years old. $51 \%$ of the sample were female who answered SPF24 and the Community Resilience Scale (Ruiz, 2015). Results: Internal reliability for full scale was 0,93, and from 0,83 to 0,86, according each scale. Factor analysis showed four main dimensions: social support, social skills, planning and goals efficacy. The first two factors correspond to the original study dimensions. In addition, a higher individual resilience was associated with higher levels of community resilience, especially with communal coping. Discussion and Conclusions: SPF-24 shows successful coefficients of internal reliability, construct validity and concurrent validity in relation to community resilience indicators. More research is required about the predictive and concurrent validity of SPF-24. 
2. Doctora en Psicología. Docente Universidad Nacional de Colombia.
Teniendo en cuenta la importancia del concepto de resiliencia como variable relevante para mitigar el impacto negativo de eventos traumáticos, es pertinente desarrollar una línea de trabajo dirigida a proporcionar instrumentos para su evaluación en poblaciones de habla hispana.
Keywords: Individual Resilience, Community Resilience, Social Support, Social Skills, Planning, Goals Efficacy.

\section{Introducción}

La resiliencia constituye un factor relevante para comprender cómo mitigan y superan el impacto de los eventos traumáticos las personas y comunidades, y a nivel individual tiene implicaciones educativas, clínicas e incluso forenses -como variable que puede ser entrenada, fomentada o evaluada-. La resiliencia comprende tanto los recursos de que disponen los individuos, familias o comunidades con anterioridad a un evento adverso y que permiten superarlo (Wexler, Moses, Hopper, Joule, \& Garouette, 2013), como el proceso de respuesta a tal adversidad y el resultado de tal respuesta, entendido como una adaptación positiva (Conger \& Conger, 2002). Otras aproximaciones son aquellas que cubren tanto las capacidades previas a la adversidad como el proceso de afrontamiento y el resultado de tal proceso (Pacheco-Mangas \& Palma-García, 2015).

Con relación a estas capacidades o recursos previos de los individuos resilientes se han identificado dimensiones y variables como la autonomía y auto-organización (Hawley, 2000; McCrea, Walton, \& Leonard, 2014), el sentido del humor (Turner, 2001; Southwick \& Charney, 2014), la autoestima positiva (Davidson, 2008; Turner, 2001) o el apoyo social de la familia y de otros (Amstrong, Birnie, Lefcovich, \& Ungar, 2005; Ponce-García, Madewell, \& Kennison, 2015; Scheible, Franks, \& Miller, 2010; Turner, 2001).

Para evaluar estos recursos, que actúan como factores protectores, se han diseñado y empleado diferentes instrumentos. La Connor-Davidson Resilience Scale (CD-RISC) (Bailey, Sharma, \& Jubin, 2013) consta de 25 ítems, ha sido traducido a varios idiomas incluyendo el español, y mide componentes como la competencia personal, la confianza en la intuición propia, la aceptación positiva del cambio, las relaciones seguras, el control y la espiritualidad. Por su lado, la Mastery Scale consta de siete ítems y se centra en el sentido de dominio, mientras que el Life Orientation Test (Ben-Zur \& Gilbar, 2011) evalúa el grado de pesimismo u optimismo ante la vida. En una línea parecida, la Child and Adolescent Functional Assessment Scale (CAFAS) arroja puntuaciones derivadas tanto de entrevistas a niños/as como a sus cuidadores y maestros (Boudine, 2011); mediante ciento sesenta ítems evalúa el ambiente de la escuela, del hogar y de la comunidad, las conductas hacia otros, el sentido del humor y manejo de las emociones, las conductas de auto-lesión, el consumo de drogas y el tipo de pensamiento (Scheibe et al., 2010). En Chile, Saavedra y Villalta (2008) desarrollaron una escala de resiliencia (Escala de Resiliencia - SV) que en una primera versión cubría factores como: autonomía, satisfacción, pragmatismo, vínculos, afectividad, autoeficacia, aprendizaje, generatividad, modelos o roles. Posteriormente, los autores han ofrecido una versión reducida de 36 ítems (Villalta \& Delgado, 2013).

Por su parte, Ponce-García y colaboradores (2015) proponen la Scale of Protective Factors-24 (SPF-24), para evaluar la presencia de Factores Protectores que promueven la resiliencia. Para su desarrollo contaron con una muestra total de 942 estudiantes divididos en tres estudios, los cuales respondieron a lo largo de dos años a una versión inicial de la escala de treinta y cinco ítems y a la versión resultante de veinticuatro. En el primer estudio, la escala total arrojó una fiabilidad interna total de 0,91, y una estructura factorial de cuatro dimensiones -apoyo social, habilidades sociales, conducta prioritaria/planificación y eficacia de metas-, con coeficientes de fiabilidad interna entre 0,78 (eficacia de metas) y 0,93 (apoyo social). Además, los puntajes de 
Pág 3

La resiliencia comunitaria puede estar asociada con el apoyo social percibido por los individuos (Southwick \& Charney, 2014), mientras que dimensiones más cognitivas como la habilidad de planificar pueden ser más independientes del contexto comunitario, desde una perspectiva tradicional de la resiliencia como capacidad de soportar el impacto de experiencias y eventos adversos. esta versión de la SPF fueron convergentes con las respuestas de la Hospital Anxiety and Depression Scale de 24 ítems. De la depuración de reactivos, los autores extrajeron la versión de 24 ítems (SPF-24), que mostró validez concurrente con el CD-RISC y con la Resilience Scale (RS). Un análisis factorial confirmatorio halló los mismos cuatro factores encontrados en la primera muestra, agrupados a su vez en dos factores de segundo orden: habilidades interpersonales (apoyo social y confianza en habilidades sociales) y un factor cognitivo individual (conducta planificada y eficacia de metas), con los siguientes coeficientes: $X 2$ (239) $=516,34$ con $p<.001 ; C F I=0,946$, RMSEA: 0,059 y NFI: 0,906. En un trabajo posterior con 450 estudiantes con niveles autoreportados altos de estrés o traumas, de tres escuelas del suroeste de Estados Unidos, el modelo resultante arrojó un $X 2(246)=669,75$ con $p<.001 ; C F l=0,925, R M-$ SEA: 0,065 y NFI: 0,887 (Madewell \& Ponce, 2016). En este mismo trabajo, los puntajes en el SPF-24 también mostraron validez concurrente con puntajes en la RS y el CD-RISC.

Teniendo en cuenta la importancia del concepto de resiliencia como variable relevante para mitigar el impacto negativo de eventos traumáticos, es pertinente desarrollar una línea de trabajo dirigida a proporcionar instrumentos para su evaluación en poblaciones de habla hispana. Aunque existen instrumentos elaborados en español como el de Villalta y Delgado (2013) o adaptados al español, como el mencionado CD-RISC, escasean pruebas psicológicas de evaluación de la resiliencia individual con estudios psicométricos basados en muestras colombianas. Los resultados del SPF-24 (Ponce-García et al., 2015), su breve extensión y su capacidad de medir aspectos interpersonales ausentes en otras pruebas de resiliencia (Madewell \& Ponce-García, 2016), determinan el objetivo de la presente investigación: conocer el comportamiento psicométrico -fiabilidad interna, validez factorial, validez concurrente- de la SPF-24 en una muestra de personas colombianas.

\section{Método}

\section{Tipo de diseño y participantes}

Se partió de un diseño psicométrico, de manera que se aplicó la escala una sola vez a un grupo de personas para conocer propiedades de fiabilidad y validez del instrumento adaptado. Se contó con una muestra por conveniencia, por tanto, no aleatoria, de 190 sujetos. Estos contestaron la prueba en el marco de la realización de una práctica de aplicación de encuesta en una materia de psicología social impartida a estudiantes de primeros semestres de la carrera de psicología de una universidad pública de la ciudad de Bogotá, Colombia.

La media de edad de los participantes se situó en los 26,4 años (DT: 10,95) y el $76 \%$ tenía entre 18 y 30 años. El $51 \%$ de los sujetos eran mujeres. En cuanto a la carrera cursada, 34,2\% eran estudiantes de psicología, 2,3\% de ingeniería, 2,3\% de Ciencia Política, 4,9\% de Educación secundaria y 1,9\% de Trabajo Social.

\section{Instrumentos}

Escala de Factores Protectores (Scale of Protective Factors-24 -SPF-24-) de Ponce-García et al. (2015). Consta de veinticuatro ítems agrupados en cuatro dimensiones: apoyo social (e.g., Mis amigos y/o mi familia me mantienen al día con las cosas importantes), confianza en habilidades sociales (e.g., Soy bueno en socializar con gente nueva), planificación (e.g., Al trabajar en algo... puedo ver el orden en que hacer las cosas) y eficacia de metas (e.g., Confío en mi habilidad para alcanzar metas). En el Apéndice de este trabajo se presenta la escala en su totalidad. Para cada ítem la persona debe indicar 
La resiliencia comunitaria puede estar asociada con el apoyo social percibido por los individuos (Southwick \& Charney, 2014), mientras que dimensiones más cognitivas como la habilidad de planificar pueden ser más independientes del contexto comunitario, desde una perspectiva tradicional de la resiliencia como capacidad de soportar el impacto de experiencias y eventos adversos. su grado de acuerdo, en una escala de siete opciones, desde "Completo desacuerdo" a "Completo acuerdo", de manera que un mayor puntaje indica mayor resiliencia de la persona, en el conjunto de la escala y por secciones. La escala mostró índices de fiabilidad interna satisfactorios en el estudio de Ponce-García et al. (2015) de 0,94 para la escala total y de 0,83 para eficacia de metas, 0,89 para habilidades sociales, 0,90 para planificación y 0,93 para apoyo social. Además, la escala ha mostrado validez concurrente con otras medidas de resiliencia como la CD-RICS y la RS. En cuanto a la validez convergente, puntuaciones altas en el SPF-24 correlacionaron significativamente con puntuaciones bajas en ansiedad y depresión. Por subescalas, todas se asociaron teórica y significativamente $(p<.001)$ con la sintomatología de ansiedad y depresión, sobre todo la planificación y el apoyo social. Y a nivel de las propias dimensiones del SPF-24, la eficacia de metas mostró las correlaciones más fuertes con las otras tres subescalas. Los puntajes en las subescalas y el puntaje total se calculan sumando los ítems correspondientes.

Escala de Resiliencia Comunitaria -ERC- (Ruiz, 2015).

Este instrumento se escogió para contrastar la validez concurrente de la escala SPF-24. Consta de 14 ítems, con cuatro opciones de respuesta, desde 1 o "Total desacuerdo" a 4 "Total acuerdo". Mayores puntajes indican percepción de más resiliencia comunitaria y de sus componentes. Un análisis factorial exploratorio encontró en una muestra colombiana dos ejes: Afrontamiento comunitario y Estima colectiva. Sin embargo, en una muestra mexicana, la escala arrojó un tercer factor denominado "Humor y creatividad" y que corresponde al marco teórico en el que se basó la construcción de la Escala (Suárez-Ojeda, 2001). La Escala mostró una fiabilidad interna satisfactoria de entre 0'87 (Ruiz, 2015) y 0,88 (Ruiz, Chan, Morales, \& Reyes, en prensa), y validez convergente con medidas de criminalidad, de forma que regiones con altas tasas de homicidios, tráfico de drogas o porte ilegal de armas presentan bajas tasas de resiliencia comunitaria.

La resiliencia comunitaria puede estar relacionada con algunas, pero no con todas las dimensiones del SPF-24. Por ejemplo, la resiliencia comunitaria puede estar asociada con el apoyo social percibido por los individuos (Southwick \& Charney, 2014), mientras que dimensiones más cognitivas como la habilidad de planificar pueden ser más independientes del contexto comunitario, desde una perspectiva tradicional de la resiliencia como capacidad de soportar el impacto de experiencias y eventos adversos. Los puntajes en las subescalas y el puntaje total se calculan sumando los ítems correspondientes (Ruiz, 2016).

\section{Datos sociodemográficos}

Se recogió información sobre la edad, el sexo y el nivel educativo de cada persona.

Análisis de datos

En primer lugar, mediante el software SPSS v. 18, se llevó a cabo el cálculo de estadísticos descriptivos de la Escala y los valores correspondientes a su fiabilidad interna, el índice de homogeneidad y la fiabilidad interna de la Escala extrayendo cada ítem. El mismo procedimiento se realizó para cada una de las subescalas teóricas del SPF-24.

En segundo lugar, se realizó un análisis factorial exploratorio de la escala, para contrastar la estructura factorial resultante con la propuesta en el estudio original. Seguidamente, se llevaron a cabo los análisis de correlaciones de Pearson entre las dimensiones del SPF-24 y las de la Escala de Resiliencia Comunitaria. 
Consideraciones bioéticas

Se tuvo en cuenta los lineamientos de la Ley colombiana 1090 de 2004 y el Código Bioético asociado, de manera que la aplicación de la batería de pruebas y el cuestionario demográfico se realizó respetando los principios de voluntariedad, anonimato y confidencialidad -al no recoger datos de identificación de la persona encuestada como su nombre o apellidos, o el número del documento de identidad-, y los principios de beneficiencia y no maleficiencia. Todo ello se plasmó en un formato de consentimiento informado insertado al comienzo de la batería, que informaba del propósito general de la investigación, y del carácter voluntario de su participación en la misma. Los datos fueron recogidos a través de una actividad con estudiantes de una materia universitaria de Psicología social.

\section{Resultados}

La fiabilidad interna del conjunto de la SPF-24 fue de $\alpha$ de Cronbach: 0,91, con índices de homogeneidad ítem-escala total entre $r(184)=0,285$ y 0,625. Por su lado, la ERC arrojó una fiabilidad interna de $\alpha: 0,85$, para los catorce ítems, con índices de homogeneidad entre $r(180)=0,315$ y 0,665, excepto para el ítem 10, con $r=0,138$ (ver Tabla 1).

Tabla 1. Indices de fiabilidad interna de las subescalas del SPF-24 y de la Escala de Resiliencia Comunitaria

\begin{tabular}{lcccc}
\hline \multicolumn{1}{c}{ Dimensiones SPF-24 } & Media y DT & Indice de Homogeneidad & Variación $\boldsymbol{\alpha}$ Cronbach & $\boldsymbol{\alpha}$ de Cronbach \\
\hline Apoyo social & $29,37(6,52)$ & $0,410-0,754$ & $0,781-0,848$ & 0,84 \\
Habilidades sociales & $29,53(7,56)$ & $0,543-0,823$ & $0,864-0,904$ & 0,90 \\
Planificación & $30,52(6,49)$ & $0,464-0,708$ & $0,781-0,827$ & 0,83 \\
Eficacia de metas & $31,29(5,59)$ & $0,632-0,697$ & $0,834-0,846$ & 0,86 \\
\hline Dimensiones de la ERC & $\mathbf{M e d i a} \boldsymbol{y} \boldsymbol{D T}$ & Indice de Homogeneidad & Variación $\boldsymbol{\alpha}$ Cronbach & $\boldsymbol{\alpha}$ de Cronbach \\
\hline Afrontamiento comunal & $26,96(5,54)$ & $0,132-0,649$ & $0,765-0,819$ & 0,81 \\
Estima colectiva & $6,61(2,57)$ & $0,338-0,629$ & $0,453-0,644$ & 0,662 \\
\hline
\end{tabular}

A partir del análisis de distribución de las puntuaciones y de acuerdo con los centiles 10 y 90 , se pueden identificar los puntos de corte para el $10 \%$ de puntuaciones más bajas y más altas, respectivamente. La Tabla 2 recoge estos puntos de corte, diferenciados para los hombres y las mujeres de la muestra.

Tabla 2. Puntos de corte orientativos sobre el centil menor o igual al $10 \%$ de puntuaciones más bajas y el centil igual o mayor al $10 \%$ de puntuaciones más altas

\begin{tabular}{lccc}
\hline \multicolumn{2}{c}{ Puntos de corte } & Puntajes bajos (centil del 10\%) & Puntajes altos (centil del 90\%) \\
\hline \multirow{2}{*}{ Apoyo social } & Hombres & 18 & 37 \\
& Mujeres & 19 & 36 \\
\hline \multirow{2}{*}{ Habilidades sociales } & Hombres & 19 & 40 \\
\cline { 2 - 4 } & Mujeres & 20 & 39 \\
\hline \multirow{2}{*}{ Planificación } & Hombres & 26 & 41 \\
\cline { 2 - 4 } & Mujeres & 22 & 38 \\
\hline \multirow{2}{*}{ Eficacia de metas } & Hombres & 27 & 38 \\
\cline { 2 - 4 } & Mujeres & 25 & 39 \\
\hline \multirow{2}{*}{ SPF-24 (total) } & Hombres & 95 & 150 \\
& Mujeres & 96 & 143 \\
\hline
\end{tabular}


Por otro lado, un análisis factorial de máxima verosimilitud y rotación promax arrojó seis factores, de los cuales los cuatro primeros presentan autovalores por encima de 1 , explicando el $54,30 \%$ de la varianza. Los cuatro factores hallados corresponden, respecto a la estructura factorial original (Ponce et al., 2015), al segundo factor (habilidades sociales), al primero (apoyo social), parcialmente al tercero (planificación) y parcialmente al cuarto factor original (eficacia). Dos ítems del cuarto factor original constituyen el quinto factor del presente estudio y dos ítems del tercer factor original constituyen el sexto factor en el mismo. Previamente al análisis factorial, el índice KMO obtenido fue de 0,880 y el coeficiente de esfericidad de Barlett de 2,566, con $p<.001$ (ver Tabla 3).

Tabla 3. Análisis factorial máxima verosimilitud y matriz rotada de la SPF-24

\begin{tabular}{|c|c|c|c|c|c|c|}
\hline ITEM & Factor 1 & Factor 2 & Factor 3 & Factor 4 & Factor 5 & Factor 6 \\
\hline SPF08 & 0,946 & & & & & \\
\hline SPF07 & 0,898 & & & & & \\
\hline SPF12 & 0,752 & & & & & \\
\hline SPF09 & 0,743 & & & & & \\
\hline SPF10 & 0,729 & & & & & \\
\hline SPF11 & 0,487 & & & & & \\
\hline SPF04 & & 0,964 & & & & \\
\hline SPF03 & & 0,922 & & & & \\
\hline SPF06 & & 0,620 & & & & \\
\hline SPF01 & & 0,561 & & & & \\
\hline SPF05 & & 0,506 & & & & \\
\hline SPF02 & & 0,322 & & & & \\
\hline SPF15 & & & 0,789 & & & \\
\hline SPF13 & & & 0,660 & & & \\
\hline SPF16 & & & 0,660 & & & \\
\hline SPF14 & & & 0,625 & & & \\
\hline SPF21 & & & & 0,597 & & \\
\hline SPF19 & & & & 0,580 & & \\
\hline SPF20 & & & & 0,573 & & \\
\hline SPF22 & & & & 0,524 & & \\
\hline SPF24 & & & & & 0,835 & \\
\hline SPF23 & & & & 0,326 & 0,772 & \\
\hline SPF17 & & & & & & 0,752 \\
\hline SPF18 & & & 0,347 & & & 0,348 \\
\hline Autovalor: & 7,29 & 2,38 & 1,75 & 1,08 & 0,76 & 0,58 \\
\hline$\%$ Varianza & 32,59 & 9,93 & 7,28 & 4,50 & 3,16 & 2,43 \\
\hline
\end{tabular}

Por último, se calcularon correlaciones entre las dimensiones del SPF-24 y las de la ERC con el fin de explorar la validez concurrente del SPF-24. Se aprecia por un lado correlaciones muy significativas entre las dimensiones de cada una de estas escalas. En segundo lugar, en cuanto a las relaciones entre los dos instrumentos, se hallan correlaciones significativas, aunque moderadas en cuanto al tamaño del coeficiente de correlación entre el puntaje total en el SPF y sus subescalas con la 
puntuación total en Resiliencia comunitaria y con la subescala de afrontamiento comunitario. Respecto a la estima colectiva, las correlaciones son nulas o escasas con las dimensiones del SPF-24 (ver Tabla 4).

Tabla 4. Correlaciones de Pearson entre los indicadores del SPF24 y los de resiliencia comunitaria

\begin{tabular}{|c|c|c|c|c|c|c|c|}
\hline Variables & 2 & 3 & 4 & 5 & 6 & 7 & 8 \\
\hline 1. SPF24 & $\begin{array}{c}0,701 \\
* * *\end{array}$ & $\underset{* * *}{0,737}$ & 0,804 & 0,796 & $\begin{array}{c}0,299 \\
* * *\end{array}$ & $\underset{* * *}{0,317}$ & $\begin{array}{c}0,178 \\
*\end{array}$ \\
\hline 2. Apoyo & 1,000 & .293 & 0,393 & 0,674 & $\underset{* * *}{0,300}$ & $\underset{* * *}{0,296}$ & $\underset{* * *}{0,232}$ \\
\hline 3. Habilidades Sociales & & 1,000 & $\begin{array}{c}0,448 \\
* * * \\
\end{array}$ & $\begin{array}{c}0,694 \\
* * *\end{array}$ & $0,125+$ & $\begin{array}{c}0,143 \\
*\end{array}$ & $\begin{array}{c}0,044 \\
n, 5\end{array}$ \\
\hline 4. Planificación & & & 1,000 & $\underset{* * *}{0,801}$ & $\underset{* *}{0,233}$ & $\begin{array}{c}0,253 \\
* * *\end{array}$ & $0,124+$ \\
\hline 5. Eficacia de metas & & & & 1,000 & $\underset{* * *}{0,300}$ & $0,0,321$ & $\begin{array}{c}0,179 \\
*\end{array}$ \\
\hline 6. Resiliencia comunitaria & & & & & 1,000 & 0,964 & 0,818 \\
\hline $\begin{array}{l}\text { 7. Afrontamiento } \\
\text { Comunitario }\end{array}$ & & & & & & 1,000 & $\underset{* * *}{0,635}$ \\
\hline 8.Estima colectiva & & & & & & & 1,000 \\
\hline
\end{tabular}

\section{Discusión y Conclusiones}

Frente a perspectivas sobre la resiliencia centradas exclusivamente en indagar acerca de los factores individuales que la caracterizan, otras aproximaciones más recientes evalúan la interacción entre aquellos factores y otros de corte psicosocial, como el apoyo social (Amstrong et al., 2005; Conger \& Conger, 2002) o la conexión con recursos familiares comunitarios (Gray, Mendelshon, \& Omoto, 2015; Walsh, 2007). En este orden de cosas, el interés del SPF-24 (Ponce-García et al., 2015) radica en combinar la inclusión en sus componentes de dimensiones como el apoyo social con factores más individuales como las habilidades sociales, la planificación y la confianza en el logro de objetivos propios (eficacia de metas), en un número de ítems relativamente breve.

En este primer abordaje de su comportamiento psicométrico en población colombiana, el SPF-24 arroja unos índices psicométricos de fiabilidad interna aceptables, tanto para el total de la Escala como para cada uno de sus componentes teóricos. Ello se complementa con una estructura de correlaciones entre cada ítem y su subescala francamente alta, de al menos $r$ : 0,410.

En segundo lugar, la adaptación al español muestra una estructura factorial coincidente a grandes rasgos con la del estudio original, con cuatro factores principales que se corresponden en su contenido con los propuestos por los autores en su estudio original. En el presente caso, el orden de los factores hace referencia a las habilidades sociales, el apoyo social de familia y amigos, la capacidad de planificación y la eficacia de metas. Se recomienda, para un análisis posterior, analizar si esta estructura factorial se confirma en un estudio que cuente con una muestra más 
La estima colectiva percibida no guarda relación con el apoyo percibido ni con las otras dimensiones de resiliencia individual, pero sí con el afrontamiento comunitario: percibir en la comunidad estrategias de afrontamiento de los problemas comunes puede proveer modelos de técnicas y de actitudes hacia la resolución de los problemas, objetivos y desafíos individuales. amplia, de forma que los cuatro ejes se diferencien de forma nítida en lugar de las seis dimensiones que se muestran en el presente trabajo.

En tercer lugar, a nivel de correlaciones se hallaron relaciones significativas y coherentes entre las cuatro dimensiones del SPF-24, como cabría esperar a partir de los coeficientes de consistencia interna elevados. Ahora bien, aunque todas las correlaciones son significativas, las más fuertes son las que se presentan entre la dimensión de eficacia y las demás, mientras que la correlación menor se da entre las dimensiones de apoyo percibido y de habilidades sociales. De esta manera, puede postularse que la eficacia de metas autopercibida se nutre tanto de un ambiente que apoya como de capacidades percibidas, por ejemplo, la de planificar y las habilidades sociales. En esta misma línea, subrayando la importancia del contexto sociocomunitario como lo hacen Jackson, Wolven y Aguilera (2013) o Shacham (2015), se hallaron relaciones directas y coherentes entre la resiliencia individual, medida con el SPF-24, con la resiliencia percibida en la comunidad, pero especialmente con el afrontamiento comunitario. Es decir, como cabría esperar, la estima colectiva percibida no guarda relación con el apoyo percibido ni con las otras dimensiones de resiliencia individual, pero sí con el afrontamiento comunitario: percibir en la comunidad estrategias de afrontamiento de los problemas comunes puede proveer modelos de técnicas y de actitudes hacia la resolución de los problemas, objetivos y desafíos individuales, probablemente con la mediación del entorno familiar y escolar, aunque estos últimos aspectos no han sido objeto de abordaje en el presente estudio. De esta manera, las relaciones encontradas en el presente estudio entre resiliencia comunitaria y resiliencia individual pueden dar apoyo para un modelo que articule la influencia del nivel comunitario sobre el individual, en la medida en que especialmente el afrontamiento comunitario -es decir, las percepciones que los individuos tienen acerca de cómo sus comunidades afrontan los problemas y retos colectivos- se asocia con una percepción más positiva de las habilidades propias de tipo social, de planificación y de logro. Así pues, desde esta perspectiva, la existencia de resiliencia comunitaria podría contribuir a fomentar la resiliencia individual-mientras que lo inverso no necesariamente se daría-, quizá vía la actuación de modelos que proporcionan estrategias útiles para la resolución de problemas.

Ahora bien, otra interpretación de estos resultados es que los sujetos que se perciben a sí mismos con mejor resiliencia de alguna manera también perciben mejor la resiliencia de sus comunidades. Sin embargo, esta interpretación no es del todo satisfactoria con relación a los resultados que proporcionan los análisis, en el sentido de que no se encontraron correlaciones fuertes y estables entre las distintas dimensiones del SPF-24 y la estima colectiva de la ERC. De hecho, la correlación más fuerte con la estima colectiva y el SPF-24 fue entre la dimensión más "social" del SPF-24, es decir, la percepción de apoyo social.

Debido al carácter no representativo de la muestra y a la no inclusión de otros instrumentos sobre resiliencia individual en el presente estudio, queda para futuros trabajos en muestras iberoamericanas conocer la validez concurrente del SPF-24 con otras medidas de resiliencia individual, y profundizar el análisis de su validez predictiva con resultados en medidas de desempeño escolar, de sintomatología psicológica o de conductas prosociales o de riesgo; ello en muestras de mayor representatividad que la obtenida para este estudio y de grupos específicos, como niños/as y adolescentes. En este sentido, los puntos de corte proporcionados en este trabajo se ofrecen por su valor orientativo y deben ser tomados con precaución a la espera de estudios específicos con esos otros grupos poblacionales. Por el mo- 
mento, el SPF-24, a la luz de los resultados arrojados hasta el momento mantiene su interés como una medida breve de factores relevantes de resiliencia individual.

\section{Referencias}

Amstrong, M.I., Birnie- Lefcovitch, S., \& Ungar, M. T. (2005). Pathways between social support, family well-being, quality of parenting, and child resilience: what we know. Journal of Child and Family Studies, 14(2), 269-281. doi: http://dx.doi. org/10.1007/s10826-005-5054-4

Bailey, A., Sharma, M., \& Jubin, M. (2013). The mediating role of social support, cognitive appraisal, and quality health care in black mothers' stress-resilience process following loss to gun violence. Violence and Victims. 28(2), 233-247. doi: http://dx. doi.org/10.1891/0886-6708.11-00151

Bez-Zur, H., \& Gilbar, O. (2011). Resilience and Distress: Israelis Respond to the Disengagement from Gaza and the Second Lebanese War Community Mental Health Journal, 47:551-559. doi http://dx.doi.org/10.1007/s10597-011-9371-3

Boudine, M.N. (2011). The Child and Adolescent Functional Assessment Scale (CAFAS): a reliability and validity evaluation. Theses and Dissertations, Paper 534. Toledo: The University of Toledo.

Conger, R.D., \& Conger, K. J. (2002). Resilience in Midwestern Families: Selected Findings from the First Decade of a Prospective, Longitudinal Study. Journal of Marriage and Family, 64(2), 361- 373.

Davidson, R. (2008). More than 'Just Coping': The Antecedents and dynamics of resilience in a qualitative longitudinal study. Social Policy \& Society, 8(1), 115-125.

Gray, N.N., Mendelsohn, D.M., \& Omoto, A.M. (2015). Community connectedness, challenges, and resilience among gay latino immigrants. American Journal of Community Psychology, 55, 202-214. doi: http://dx.doi.org/10.1007/s10464-0149697-4

Hawley, D. R. (2000). Clinical implications of family resilience. The American Journal of Family Therapy, 28(2), 101-116. doi: http://dx.doi.org/10.1080/019261800261699

Jackson, K. F., Wolven, T., \& Aguilera, K. (2013). Mixed Resilience: A study of multiethnic Mexican American stress and coping in Arizona. Family relations, 62(1), 212225. doi: http://dx.doi.org/10.1111/j.1741-3729.2012.00755.x

Madewell, A.N., \& Ponce-García, E. (2016). Assessing resilience in emerging adulthood: The Resilience Scale (RS), Connor-Davidson Resilience Scale (CD-RISC), and Scale of Protective Factors (SPF). Personality and Individual Differences, 97, 249-255. doi: http://dx.doi.org/10.1016/j.paid.2016.03.036

McCrea, R., Walton, A., \& Leonardo, R. (2014). A conceptual framework for investigating community wellbeing and resilience. Rural society, 23(3), 270-282. doi: http://dx.doi. org/10.1080/10371656.2014.11082070

Pacheco-Mangas, J., \& Palma-Garcia, M. O. (2015). La resiliencia en Servicios Sociales Comunitarios: un abordaje desde la perspectiva de los profesionales. Revista Internacional de Trabajo Social y Bienestar, 4, 29-38

Ponce - Garcia, E., Madewell, A. N., \& Kennison; S.M. (2015). The Development of the Scale of Protective Factors: Resilience in a Violent Trauma Sample. Violence and Victims, 30(5) 735- 755. doi: http://dx.doi.org/10.1891/0886-6708.VV-D-1400163

Ruiz, J.I. (2015). Resiliencia comunitaria: propuesta de una escala y su relación con indicadores de violencia criminal. Pensamiento Psicológico, 13(1), 119-135.

Ruiz, J.I. (2016). Tejido social, violencia criminal y percepción de inseguridad. En L.A. Rodríguez-Cey (Ed.). Psicología Jurídica y Forense: Debates, propuestas e investigaciones (61-90). Madrid: EOS. 
Ruiz, J.I., Chan, C., Morales, L.A., \& Reyes, H. (en prensa). Scale of community resilience: analysis of psychometric aspects and relations with criminal victimization in Mexican university students. Revista Mexicana de Psicología.

Saavedra, E., \& Villalta, M. (2008). Escala de Resiliencia SV-RES. Santiago de Chile-CEANIM.

Scheible, J. L., Franks, B. A., \& Miller, M. D. (2010). Emotion dysregulation and academic resilience in maltreated children. Child Youth Care Forum, 39, 289-303. doi: http://dx.doi.org/10.1007/s10566-010-9105-7

Shacham, M. (2015). Suddenly - war. Intervention program for Enhancing teachers and children's resilience Following war. Revista de Cercetare si Interventie Social. 48, 60-68.

Southwick, S.M., \& Charney, D.S. (2014). Resilence: the science of mastering life's greatest challenges. Nueva York, NY: Cambridge University Press.

Suárez-Ojeda, E. N. (2001). Una concepción latinoamericana: la resiliencia comunitaria. En A. Melillo, E. N. Suárez-Ojeda (Coord.). Resiliencia: descubriendo las propias fortalezas 67-82). Buenos Aires: Paidós.

Turner, S. G. (2001) Resilience and social work practice: Three case studies. Families in Society, 82(5), 441-448. doi: http://dx.doi.org/10.1606/1044-3894.176

Villalta, M.A., \& Delgado, A. (2013). Propuesta de una escala para resiliencia para adolescentes chilenos: SV-RES abreviada. En J. Ma. Madariaga, A. Forés, E. Goñi, M. de O. Palma, O. Pérez-Muga, y otros. La construcción social de la resiliencia (2129). Bilbao: Universidad del País Vasco.

Walsh, F. (2007). Traumatic loss and Major Disasters: Strengthening family and community resilience. Family Process, 46(2), 207-227.

Wexler, L., Moses, J., Hopper, K., Joule, L., Garoutte, J., \& LCS CIPA Team (2013). Central role of relatedness in Alaska native youth resilience: Preliminary themes from one site of the circumpolar indigenous pathways to adulthood (CIPA) Study. American Journal of Community Psychology, 52, 393-405. doi: http://dx.doi. org/10.1007/s10464-013-9605-3 


\section{APÉNDICE}

\section{SPF-24 versión española}

(Ponce-García, Madewell y Kennison, 2015, adaptado por Ruiz y Herrera)

Por favor, indique su grado de acuerdo respecto a cada una de las siguientes frases, según usted percibe a su familia y amigos y a usted mismo.

\begin{tabular}{|c|c|c|c|c|c|c|c|}
\hline Mis amigos y/o mi familia & $\begin{array}{l}\text { Completo } \\
\text { desacuerdo }\end{array}$ & & & & & & $\begin{array}{l}\text { Completo } \\
\text { acuerdo }\end{array}$ \\
\hline Me mantienen al día con las cosas importantes & 1 & 2 & 3 & 4 & 5 & 6 & 7 \\
\hline Ven las cosas de la misma manera & 1 & 2 & 3 & 4 & 5 & 6 & 7 \\
\hline Son unidos & 1 & 2 & 3 & 4 & 5 & 6 & 7 \\
\hline Se apoyan mutuamente & 1 & 2 & 3 & 4 & 5 & 6 & 7 \\
\hline Son optimistas & 1 & 2 & 3 & 4 & 5 & 6 & 7 \\
\hline Pasan tiempo libre juntos & 1 & 2 & 3 & 4 & 5 & 6 & 7 \\
\hline Soy bueno en & $\begin{array}{l}\text { Completo } \\
\text { desacuerdo }\end{array}$ & & & & & & $\begin{array}{l}\text { Completo } \\
\text { acuerdo }\end{array}$ \\
\hline Socializar con gente nueva & 1 & 2 & 3 & 4 & 5 & 6 & 7 \\
\hline Relacionarme con otros & 1 & 2 & 3 & 4 & 5 & 6 & 7 \\
\hline Hacer nuevos amigos & 1 & 2 & 3 & 4 & 5 & 6 & 7 \\
\hline Estar con otras personas & 1 & 2 & 3 & 4 & 5 & 6 & 7 \\
\hline Trabajar con otros como parte de un equipo & 1 & 2 & 3 & 4 & 5 & 6 & 7 \\
\hline Iniciar nuevas conversaciones & 1 & 2 & 3 & 4 & 5 & 6 & 7 \\
\hline Al trabajar en algo & $\begin{array}{l}\text { Completo } \\
\text { desacuerdo }\end{array}$ & & & & & & $\begin{array}{l}\text { Completo } \\
\text { acuerdo }\end{array}$ \\
\hline Puedo ver el orden en qué hacer las cosas & 1 & 2 & 3 & 4 & 5 & 6 & 7 \\
\hline Planifico las cosas & 1 & 2 & 3 & 4 & 5 & 6 & 7 \\
\hline Organizo bien mi tiempo & 1 & 2 & 3 & 4 & 5 & 6 & 7 \\
\hline Establezco prioridades antes de comenzar & 1 & 2 & 3 & 4 & 5 & 6 & 7 \\
\hline Lo hago mejor si establezco objetivos & 1 & 2 & 3 & 4 & 5 & 6 & 7 \\
\hline $\begin{array}{l}\text { Hago una lista de cosas para hacer, en orden de } \\
\text { importancia }\end{array}$ & 1 & 2 & 3 & 4 & 5 & 6 & 7 \\
\hline Confío en mi habilidad para & $\begin{array}{l}\text { Completo } \\
\text { desacuerdo }\end{array}$ & & & & & & $\begin{array}{l}\text { Completo } \\
\text { acuerdo }\end{array}$ \\
\hline Alcanzar metas & 1 & 2 & 3 & 4 & 5 & 6 & 7 \\
\hline Pensar y planificar & 1 & 2 & 3 & 4 & 5 & 6 & 7 \\
\hline Hacer buenas decisiones/elecciones & 1 & 2 & 3 & 4 & 5 & 6 & 7 \\
\hline Reaccionar con rapidez & 1 & 2 & 3 & 4 & 5 & 6 & 7 \\
\hline Tener éxito & 1 & 2 & 3 & 4 & 5 & 6 & 7 \\
\hline Resolver problemas & 1 & 2 & 3 & 4 & 5 & 6 & 7 \\
\hline
\end{tabular}

